\title{
Asymmetric partially coherent solitons in saturable nonlinear media
}

\author{
Natalia M. Litchinitser, ${ }^{1}$ Wiesław Królikowski, ${ }^{2}$ Nail N. Akhmediev, ${ }^{3}$ and Govind P. Agrawal ${ }^{1}$ \\ ${ }^{1}$ The Institute of Optics and Rochester Theory Center of Optical Sciences and Engineering, University of Rochester, \\ Rochester, New York 14627 \\ ${ }^{2}$ Australian Photonics CRC, Laser Physics Centre, Research School of Physical Sciences and Engineering, \\ The Australian National University, Canberra, Australian Capital Territory 0200, Australia \\ ${ }^{3}$ Australian Photonics CRC, Optical Sciences Centre, Research School of Physical Sciences and Engineering, \\ The Australian National University, Canberra, Australian Capital Territory 0200, Australia
}

(Received 20 January 1999)

\begin{abstract}
We investigate theoretically properties of partially coherent solitons in optical nonlinear media with slow saturable nonlinearity. We have found numerically that such a medium can support spatial solitons which are asymmetric in shape and are composed of only a finite number of modes associated with the self-induced waveguide. It is shown that these asymmetric spatial solitons can propagate many diffraction lengths without changes, but that collisions change their shape and may split them apart. [S1063-651X(99)12808-3]
\end{abstract}

PACS number(s): 42.65.Tg, 41.20.Jb

Incoherent spatial solitons have attracted considerable attention recently [1-7], especially after the first experimental observation of partially coherent solitons (PCS's) was made by Mitchell et al. [8]. The case of temporally incoherent solitons was considered previously by Hasegawa in a series of papers [9], both for waves in bulk dispersive media and for nonlinear pulses in multimode fibers. In optical fibers, the wave packet can propagate as a single stationary pulse when the intermodal dispersion is balanced by the nonlinearity. Intermodal dispersion usually exceeds the intramodal dispersion and, as a result, the generation of incoherent solitons in optical fibers requires unrealistically high pulse energies. The situation with spatial solitons in this respect might be different. Photorefractive materials are probably the most suitable medium for experimental studies, since they generally exhibit very strong nonlinear effects at extremely low optical powers [10-12].

Spatial incoherent solitons are objects qualitatively different from temporal incoherent solitons. A general description of incoherent spatial beam propagation can be quite complicated $[13,14]$, and meets with serious mathematical difficulties. Nevertheless, the case of incoherent spatial solitons is a special one. From a conceptual point of view, for spatial solitons, it is the diffraction of the beam components which has to be balanced by the nonlinearity. This basic principle, which is similar to the one for fundamental solitons, is also valid for incoherent solitons. Using this principle allows for great simplifications. In fact, several different approaches have been used for investigating incoherent solitons theoretically. The "coherent-density approach,"' in which a partially coherent beam is represented as a superposition of mutually incoherent components, was developed by Christodoulides and co-workers $[1,2]$. For the special case of the logarithmic nonlinearity, symmetric solutions can be written in an analytic form [1].

The description of optical beams in nonlinear media in terms of a self-induced multimode waveguide has been especially fruitful. This idea has been suggested for higher order solitons in birefringent media $[15,16]$ and was used recently for incoherent solitons $[3-6,17]$. In this point of view, stationary soliton propagation is governed by a proper combination of various mutually incoherent linear modes of the self-induced waveguide. On the other hand, this approach has so far provided only symmetric solutions $[1,2,6,7]$, although asymmetric solitons are likely to exist. For the Kerrlike medium, asymmetric solutions were recently considered in Ref. [18]. In this special case the problem is integrable, and all solutions including asymmetric ones can be written in analytical form. The analysis of Ref. [18] showed that PCS's can be considered from two complementary point of views. On the one hand, the PCS is a multimoded self-induced waveguide and, on the other hand, it is a multisoliton complex. This latter point of view is another facet of PCS's which allows one to understand in simple terms such complicated phenomena as collision of PCS's.

One of the remarkable features of integrable systems is that there is no binding energy between the fundamental solitons. In particular, for the system considered in Ref. [18], there is no binding energy between the fundamental solitons in the multisoliton complex, so that they may be located arbitrarily inside the PCS. The arbitrariness in amplitudes and locations allows the whole PCS to be variable in shape.

On the other hand, it is known that for saturable medium the binding energy between the fundamental solitons is not zero, and the question of the existence of a PCS with a variable shape becomes questionable. The diffractionless rayoptics limit for treating spatially incoherent solitons was proposed in Ref. [19]. This approach is accurate when the size of the PCS is much larger than the optical wavelength. In terms of a multimode waveguide, this limit is valid when the number of modes goes to infinity, so that the soliton becomes completely incoherent. It has been shown, in this limit, that solitons of arbitrary shape may exist for nonlinearities other than Kerr-like. However, the question of the existence of asymmetric solitons in media with saturable nonlinearity when the number of modes is finite is not obvious for the reasons mentioned above. Hence a separate study of this problem is needed.

In this paper, we investigate the properties of partially coherent solitons in a saturable nonlinear optical medium. 


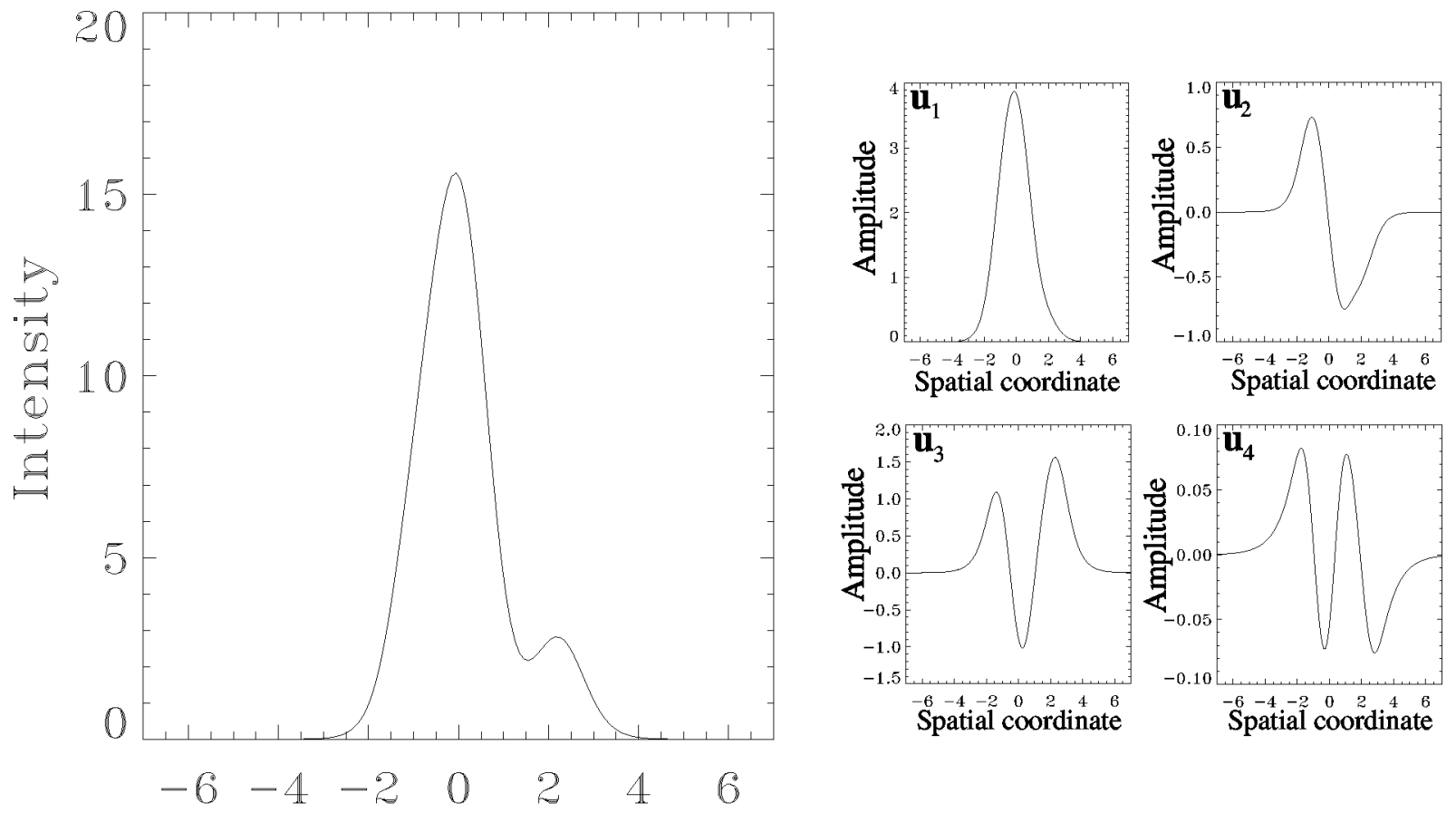

Spatial coordinate

FIG. 1. Intensity profile of an asymmetric partially coherent soliton composed of four modes. The inset shows the amplitudes of the constituent modes. The normalized propagation constants are $\lambda_{1}=3.05249, \lambda_{2}=2.084766, \lambda_{3}=1.438924$, and $\lambda_{4}=0.60524$.

We have found asymmetric solutions for such solitons. We have shown, using numerical simulations, that these solutions propagate several diffraction lengths without changes. The interaction of incoherent and partially coherent solitons is an interesting area of research, and it has only been addressed in recent papers [4,5]. Here we have studied numerically collisions of asymmetric PCS's.

It has been shown that propagation of a partially coherent beam in nonlinear media can be represented by a set of equations for the mutually incoherent components constituting the beam $[2,9]$. For a beam consisting of $N$ such components, the corresponding equations have the following form in case of saturable nonlinearity:

$$
i \frac{\partial \psi_{i}}{\partial z}+\frac{1}{2} \frac{\partial^{2} \psi_{i}}{\partial x^{2}}+\frac{\alpha I}{1+I / I_{0}} \psi_{i}=0
$$

where $\psi_{i}$ denotes the amplitude of the $i$ th component of the beam $(i=1,2, \ldots), \alpha$ represents the strength of the nonlinearity, $I_{0}$ is the saturation parameter, $x$ and $z$ denote dimensionless transverse and propagation coordinates, respectively, and

$$
I=\sum_{i=1}^{N}\left|\psi_{i}\right|^{2}
$$

is the total intensity created by all incoherent components of the light beam. The specific form $\left(1+I / I_{0}\right)^{-1}$ of the saturation of nonlinearity used in Eq. (1) holds, for instance, for a homogeneously broadened two-level system and biased photorefractive crystals; other functional forms should also exhibit similar qualitative behaviors. The nonlinear medium is assumed to have a slow response compared with the time scale over which the phases of the individual components change, so that the change of the refractive index is determined by the total intensity given by Eq. (2). It can be shown

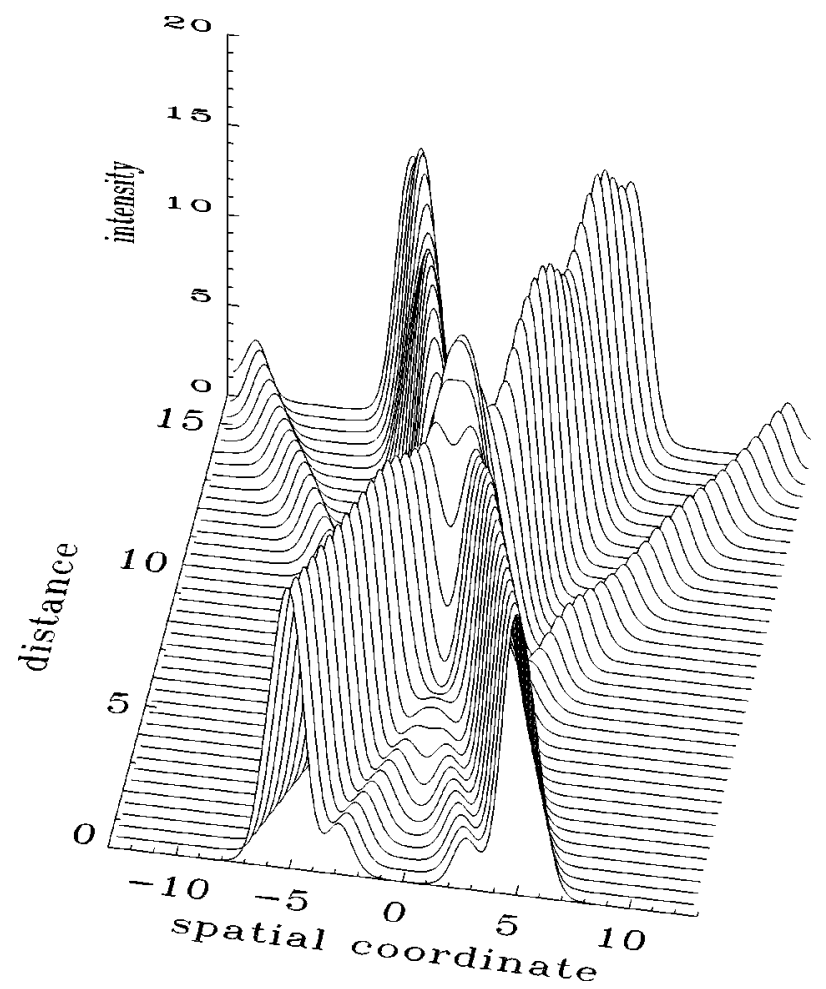

FIG. 2. Collision of the asymmetric partially coherent solitons whose intensity profile and mode amplitudes are shown in Fig. 1. The angle of collision corresponds to $\tan \theta=0.3$. 

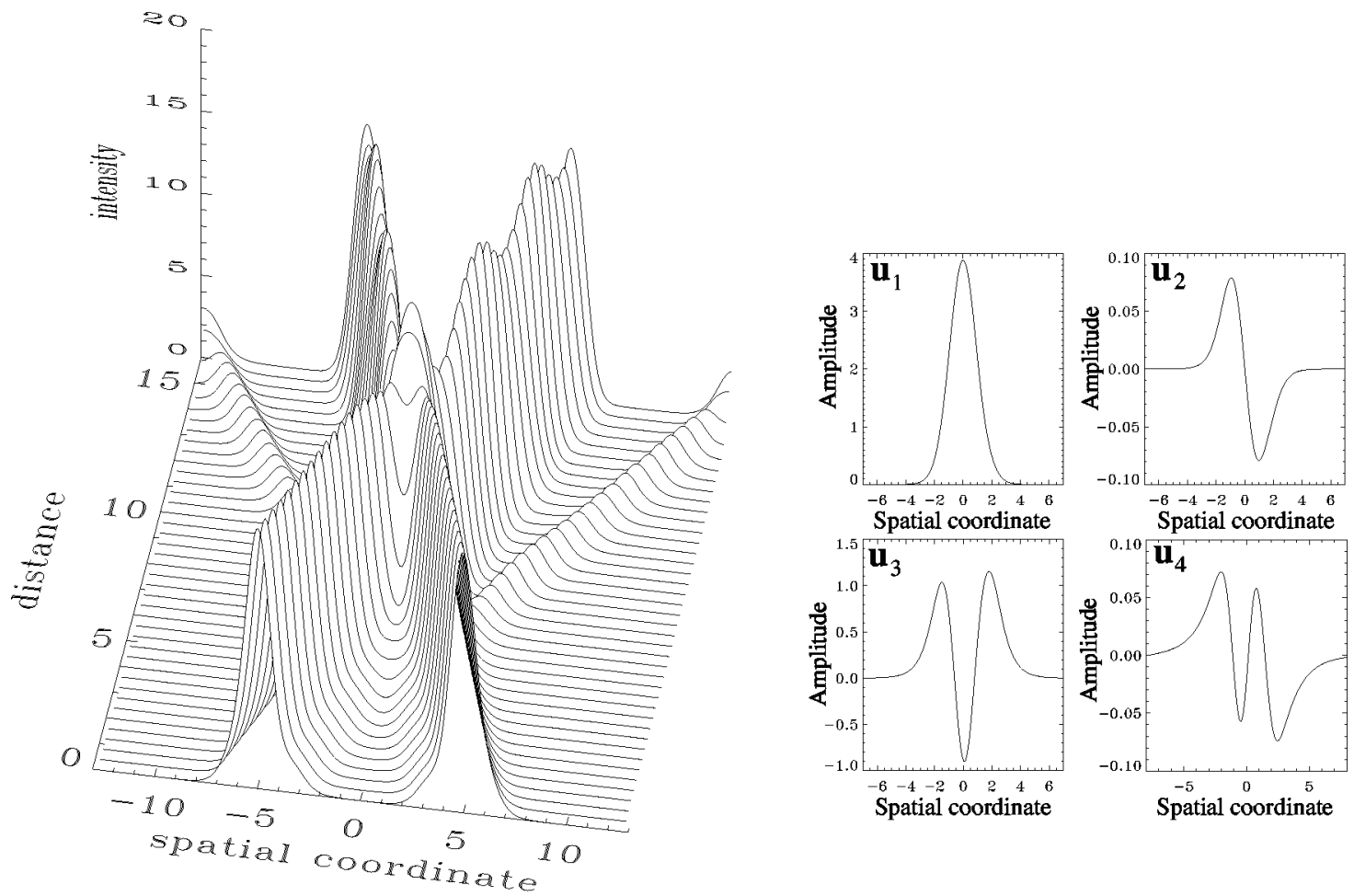

FIG. 3. Another example of collision of partially coherent solitons with $\lambda_{1}=3.000416, \lambda_{2}=1.922105, \lambda_{3}=1.002799$, and $\lambda_{4}$ $=0.25964$. The angle of collision is the same as in Fig. 2. The inset shows the amplitudes of the four constituent modes.

that the width of the spatial coherence function depends on the number of components included in the sum appearing in Eq. (2) [7]. For this reason, we refer to the beam with finite $N$ as "partially coherent."

We are interested in PCS solutions of Eq. (1) when $N$ is finite and a relatively small number. Such solutions correspond to stationary waveguides self-induced by their own modes. However, the self-consistency condition, represented by Eq. (2), requires these solutions to be multisoliton complexes, which are nonlinear superposition of fundamental solitons propagating in parallel and thus creating the waveguide. This complementary view is important for a physical understanding of the PCS's.

Stationary solutions of Eq. (1) can be written in the form

$$
\psi_{i}(x, z)=u_{i}(x) \exp \left(i \lambda_{i} z\right)
$$

with real functions $u_{i}(x)$ and real eigenvalues $\lambda_{i}$. Then the set of equations (1) reduces to a set of ordinary differential equations of the form

$$
\frac{\partial^{2} u_{i}}{\partial x^{2}}+2 \alpha\left(\frac{\sum_{j=1}^{N} u_{j}^{2}}{1+\sum_{j=1}^{N} u_{j}^{2} / I_{0}}\right) u_{i}=2 \lambda_{i} u_{i} .
$$

Physically, $\lambda_{i}$ is the propagation constant associated with the mode profile $u_{i}(x)$.

Equation (4) can be solved numerically using either a shooting or an iteration technique. In the latter case, we first find the modes of a given (zero-order approximation) waveguide, vary the amplitudes of different modes to modify the refractive index profile, and then use the self-consistency condition (2). Repeating these steps results in a convergence to a stationary beam profile. Using this iterative approach, we have found the intensity profiles of several different PCS's and their constituent linear modes. In our simulations, the propagation distance $z$ is normalized to the diffraction length, and we choose $\alpha=2$ and $I_{0}=2$ in Eq. (1). An example of a PCS is shown in Fig. 1, together with the spatial profiles of the four modes associated with the self-induced waveguide. Notice that the modes are also asymmetric since an asymmetric PCS induces index changes that are themselves asymmetric.

In turns out that, in most cases, the numerical procedure converges to a symmetric solution, similar to those presented in previous works $[3,6]$. However, some specific initial conditions with a refined iterative procedure result in asymmetric solutions such as the one displayed in Fig. 1. We should note that asymmetric solutions are generic and appear on the same basis as symmetric ones. They also appear in other nonlinear optical problems [20]. Their emergence is not completely surprising, since a symmetric nonlinear equation can have asymmetric solutions.

Note, however, that in the case of a single nonlinear Schrödinger equation (NLS) with constant coefficients, stationary asymmetric solutions do not exist. The one-soliton $(N=1)$ solution is symmetric, and all higher-order solitons are nonstationary solutions of the NLS. In fact, it appears that only a coupled set of NLS's admit stationary asymmetric solutions.

There is a deep physical reason why the symmetric solutions appear naturally. In contrast to Kerr-like media, there is a binding energy between the soliton components in saturable media. This binding energy results in an attractive force which tries to keep different components together and makes the whole soliton symmetric. This is exactly what happened in numerical simulations of Refs. [3,6]. Nevertheless, the asymmetric solutions do exist and we have found them here. 
Extensive numerical simulations indicate that asymmetric solutions are multiparameter families rather than isolated solutions. First, the mode amplitudes are variable parameters. Changing them, we were able to change the shape of the soliton continuously. Second, there must be parameters which control the asymmetry of the soliton. In the integrable case (a slow Kerr-like medium [18]), the total number of parameters which control the shape is $2 N-1$, where $N$ is the number of the modes. We can assume that PCS's in saturable media have the same property. On this basis, $N$ propagation constants are arbitrary, and $N-1$ relative parameters of asymmetry between the modes adds up to the total $2 N-1$ parameters of the solution.

At this stage of investigation, we have found several examples of asymmetric solutions, and checked that they are relatively stable solutions of Eq. (1) in the sense that they do not change their shape for several diffraction lengths during propagation inside the nonlinear media. However, the shape of the PCS changes appreciably after collisions. Moreover, the optical beam may split into several parts on collision. Two examples of collisions are shown in Figs. 2 and 3. The angle of propagation is achieved using a Galilean transformation [21] with the "velocity" $\mathrm{v}=\tan \theta$, where $\theta$ is the angle of propagation in the $x-z$ plane. As the intensity of the solitons is quite high, both cases correspond to relatively strong saturation regime $\left(I / I_{0} \approx 8\right)$. These figures show clearly that the initial soliton profiles are indeed stationary, but those which appear after collisions are not. They may oscillate and emit other solitons which propagate away from the main beam. Radiation waves also appear as a result of the collision. The nonlinearity model of the saturable medium considered in this paper is nonintegrable. Hence the soliton resulting after the collision does not have to correspond to a stationary solution [21]. The output beam generally changes on propagation (oscillates), and detailed evolution depends on the specific choice of the angle of collision and other parameters that determine the initial conditions. The oscillating beam can be considered a perturbed PCS.

In conclusion, we have shown that nonlinear optical media with slow saturable nonlinearity admit propagation of partially coherent solitons with an asymmetric intensity profile. Although such asymmetric solitons maintain their shape for several diffraction lengths if propagated in isolation, their mutual collisions generate radiation (diffractive waves), and result in a beam profile that is no longer stationary.

Natalia M. Litchinitser thanks the Aileen S. Andrew Foundation for financial support.
[1] D. N. Christodoulides, T. H. Coskun, and R. I. Joseph, Opt. Lett. 22, 1080 (1997).

[2] D. N. Christodoulides, T. H. Coskun, M. Mitchell, and M. Segev, Phys. Rev. Lett. 78, 646 (1997).

[3] M. Mitchell, M. Segev, T. Coskun, and D. N. Christodoulides, Phys. Rev. Lett. 79, 4990 (1997).

[4] V. A. Vysloukh, V. Kuznetsov, V. M. Petnikova, and V. V. Shuvalov, Kvant. Elektron. (Moscow) 24, 867 (1997) [Sov. J. Quantum Electron. 27, 843 (1997)].

[5] V. Kutuzov, V. M. Petnikova, V. V. Shuvalov, and V. A. Vysloukh, Phys. Rev. E 57, 6056 (1998).

[6] D. N. Christodoulides, T. H. Coskun, M. Mitchell, and M. Segev, Phys. Rev. Lett. 80, 2310 (1998).

[7] M. I. Carvalho, T. H. Coskun, D. N. Christodoulides, M. Mitchell, and M. Segev, Phys. Rev. E 59, 1193 (1999).

[8] M. Mitchell, Z. Chen, M. Shih, and M. Segev, Phys. Rev. Lett. 77, 490 (1996); M. Mitchell and M. Segev, Nature (London) 387, 880 (1997).

[9] A. Hasegawa, Phys. Fluids 18, 77 (1975); 20, 2155 (1977); Opt. Lett. 5, 416 (1980).

[10] G. Duree, J. L. Shultz, G. Salamo, M. Segev, A. Yariv, B. Crosignani, P. Di Porto, E. Sharp, and R. R. Neurgaonkar, Phys. Rev. Lett. 71, 533 (1993); M. Shih, M. Segev, G. C. Valley, G. Salamo, B. Crosignani, and P. Di Porto, Electron. Lett. 31, 826 (1995).
[11] M. D. Iturbe-Castillo, P. A. Marques Aguliar, J. J. SanchesMondragon, and V. Vysloukh, Appl. Phys. Lett. 64, 408 (1994).

[12] A. V. Mamaev, M. Saffman, D. Z. Anderson, and A. A. Zozulya, Phys. Rev. A 54, 870 (1996); A. A. Zozulya, D. Z. Anderson, A. V. Mamaev, and M. Saffman, Europhys. Lett. 36, 419 (1996).

[13] G. A. Pasmanik, Zh. Eksp. Teor. Fiz. 66, 490 (1974) [Sov. Phys. JETP 39, 234 (1974)].

[14] V. A. Aleshkevich, S. S. Lebedev, and A. N. Matveev, Kvant. Elektron. (Moscow) 8, 1090 (1981) [Sov. J. Quantum Electron. 11, 647 (1981)].

[15] A. Snyder, S. J. Hewlett, and D. J. Mitchell, Phys. Rev. Lett. 72, 1012 (1994).

[16] A. W. Snyder, D. J. Mitchell, and Yu. Kivshar, Mod. Phys. Lett. B 9, 1479 (1995).

[17] M. Mitchell, M. Segev, and D. N. Christodoulides, Phys. Rev. Lett. 80, 4657 (1998).

[18] N. Akhmediev, W. Królikowski, and A. W. Snyder, Phys. Rev. Lett. 81, 4632 (1998).

[19] A. W. Snyder and D. J. Mitchell, Phys. Rev. Lett. 80, 1422 (1998).

[20] N. N. Akhmediev, Zh. Eksp. Teor. Fiz. 83, 545 (1982) [Sov. Phys. JETP 56, 299 (1982)].

[21] N. Akhmediev and A. Ankiewicz, Solitons, Nonlinear Pulses and Beams (Chapman \& Hall, London, 1997). 